\title{
Entre la movilidad residencial y la migración. Factores y razones para el cambio de residencia desde un entorno urbano entre la población mayor Between Residential Mobility and Migration. Factors and Reasons for the Change of Residence from an Urban Surrounding among Old People
}

\author{
Raúl Lardiés Bosque*
}

\section{INTRODUCCIÓN}

El aumento de la movilidad residencial en el conjunto de la población, y en particular en las personas de más edad, es una característica de las sociedades desarrolladas (Módenes, 2006), de forma que cada vez más personas deciden cambiar su lugar de residencia cuando llega el momento de la jubilación. En este contexto, diversos cambios sociales, económicos y culturales han hecho que el retiro de la etapa laboral no se tenga que vivir necesariamente como una etapa de decadencia como en el pasado, sino como un período todavía de plenitud en el que las personas emprenden nuevos proyectos, incluidos los de tipo residencial (Puga, 2004a).

Cada vez es más común que las personas elijan nuevos lugares donde jubilarse, lo cual responde a múltiples factores estructurales (macro) de tipo social, económico y cultural, entre otros. Pero sólo esos factores no explican el aumento de la movilidad residencial de los mayores, sino que es necesario

\footnotetext{
* Instituto de Economía, Geografía y Demografía. Centro de Ciencias Humanas y Sociales. (CSIC) (raul.lardies@cchs.csic.es).
} 
profundizar en otras razones y motivaciones de tipo geográfico, ambiental, familiar, social, cultural, etc.

En este trabajo se analizan aquellas que han impulsado a personas mayores residentes en la ciudad de Madrid y en su área metropolitana a trasladar su lugar de residencia, en torno a la edad de jubilación, a otras zonas de España (bien de forma temporal o definitiva). El análisis de las razones se hace en relación a las principales características sociodemográficas de este grupo de población como el sexo, la edad, el estado civil, el lugar de residencia previo, la situación y la composición familiar, o el lugar de nacimiento.

Se trata de una movilidad realizada libremente, en un grupo de población todavía relativamente joven, con buena salud, y con cierto poder adquisitivo, por lo que se enmarca en la denominada movilidad residencial de amenidad (Abellán y Rojo, 1997). Sin embargo, son cambios residenciales realizados bajo formas mucho más flexibles que los que conllevaban las migraciones tradicionales, por lo que conviene hacer referencia a movilidad residencial, tal y como se comentará más adelante.

Investigar cuáles son las razones de los traslados residenciales ayudará a conocer el proceso de toma de decisión y los mecanismos subyacentes en el proceso de movilidad residencial de los mayores. Con ello se rellena el hueco de conocimiento que existe sobre el comportamiento residencial de este grupo de población, además de contribuir a la planificación de futuros lugares de retiro, de los servicios y equipamientos que tales desplazamientos requieren, y a la mejora de la calidad de vida de estas personas.

\section{EL ESTUDIO DE LAS MIGRACIONES Y LA MOVILIDAD RESIDENCIAL DE LOS MAYORES}

Dos modelos conceptuales han guiado el estudio de las migraciones (o movilidad residencial) de las personas mayores: el modelo push and pull y el de curso de vida (Walters, 2002). El primer modelo, de atracción y repulsión ha sido adoptado en numerosos estudios (Haas y Serow, 1993) y según él, las personas emigran en respuesta a las características más o menos atractivas de los lugares de origen y de destino. Puesto que se trata de un modelo de comportamiento grupal, el modelo push and pull parte de la asunción de que todas las personas prefieren el mismo tipo de características en los lugares de origen y de destino. Pero puesto que las personas mayores no son uniformes en su composición y preferencias, este modelo ha llevado a inconsistencias y a veces a resultados contradictorios (Walters, 1994). 
El segundo modelo, el de curso/ciclo de vida (life-course model), se basa en la idea de que las preferencias de los migrantes varían en función de sus atributos personales y del momento en el que se encuentran en su ciclo de vida; por ejemplo, muchos jubilados relativamente jóvenes, con buena salud e ingresos suficientes, cambian su lugar de residencia en busca de más calidad de vida y de mejores condiciones residenciales; en otros casos, quedarse viudo/a, o estar fuertemente discapacitado, genera otro tipo de traslado residencial (Conway y Houtenville, 2003). El modelo de curso de vida ha sido efectivo para mostrar cómo las tasas de movilidad varían como respuesta a los acontecimientos que se producen a lo largo de la vida, y cómo los atributos y la situación personal de los mayores condicionan su comportamiento migratorio (Puga, 2004b; Walters, 2002).

Según lo anterior, no todas las personas mayores cambian de lugar de residencia por las mismas razones, sino que varían en función de los atributos y situación particular de cada persona. Diversos autores (Litwark y Logino, 1987; Meyer y Speare, 1985) han propuesto contextos evolutivos y secuenciales de tipos de movilidad/migraciones, de forma que se tipifican, por lo general, tres grandes categorías o tipos de desplazamientos geográficos entre los mayores:

1) el primero, entre los 60 y 75 años de edad, se produce entre personas generalmente con buen estado de salud y buena situación financiera, en pareja, y por motivos relacionados con la amenidad, el coste de la vida y la búsqueda de tranquilidad.

2) el segundo movimiento, generalmente a partir de los 75 años de edad, se lleva a cabo entre personas que son viudas, que se encuentran en peor situación económica y que empiezan a necesitar algún tipo de asistencia y cuidados informales; este tipo podría ser una especie de retorno o bien al lugar previo de partida (lugar previo de residencia), o bien un desplazamiento a donde reside alguno de los hijos, familiares o amigos.

3) por último, hacia los años finales de la vida, el tercer tipo de desplazamientos es cuando la persona requiere cuidados formales, por lo que el movimiento se produce hacia una institución.

Estos tres tipos de desplazamientos no sólo están motivados por razones distintas, sino que también sus impactos (sociales, económicos, y para la persona) son diferentes. El análisis que se realiza en este artículo de las razones para el traslado residencial responde al modelo de atracción-rechazo, el cual resulta más efectivo cuando las personas (emigrantes) tienen unas mismas características y atributos (en este caso, personas que han residido 
en la misma ciudad, poseedores de residencias secundarias, y en el mismo grupo de edad); estamos, por tanto, ante el grupo de migrantes motivados por la amenidad y por la búsqueda de determinados factores ambientales (Abellán y Rojo, 1997).

Muchos de estos flujos de población mayor pueden explicarse o comprenderse a la luz de los importantes cambios sociales, culturales, económicos, tecnológicos... que se han producido en España, y en general, en los países occidentales y desarrollados. Sin embargo, estas causas explican una parte de las migraciones, pero no la parte debida a la no racionalidad y la comportamiento individual de las personas; existen otras muchas razones, personales, de tipo familiar, social, económico, que impulsan o frenan el fenómeno. Eso corrobora el hecho de que las investigaciones sobre los procesos de decisión migratoria deben conducir a modelos teóricos en los que figuren tanto los factores estructurales que determinan sus elecciones como las motivaciones de los individuos (Puga, 2004b).

\section{Conceptualización, Fuente y Metodología}

Como ya se ha señalado, desde el punto de vista conceptual parece más preciso hacer referencia a movilidad residencial en lugar de a migración, ya que el primer concepto engloba formas y tipos de desplazamientos más amplios que los estrictamente denominados como migratorios. El concepto de migración implica algo que fue común en el pasado como era el cambio del lugar de residencial habitual por otro. Ese abandono, bien fuera definitivo o temporal, suponía un cambio profundo en la vida del emigrante, puesto que le hacía romper con su vida anterior y emprender una nueva en el lugar de destino. Sin embargo, las pautas de movilidad actuales son mucho más flexibles y no siempre implican tener que abandonar necesariamente el lugar previo de residencia; se puede estar residiendo temporalmente entre dos o más lugares, compartiendo y manteniendo diferentes viviendas; por ello, más que hacer referencia a migrantes, hay que hacer, en ocasiones, a personas que se comportan como turistas (o residentes) de diferente duración, que ocupan temporalmente una vivienda secundaria (Flognfeldt, 2002). De la misma forma que muchos emigrantes internacionales viven a caballo entre dos o más países y no se ha producido el abandono del lugar previo de residencia, puesto que en muchos sentidos siguen viviendo en ambos (parte de la semana, del mes o del año), lo mismo ocurre en este tipo de migrantes mayores, al repartir su tiempo y su vida entre su residencia previa y la nueva. 
Este comportamiento dificulta de manera importante la cuantificación de estos movimientos residenciales. El objetivo de esta investigación no es esa cuantificación, pero de haberlo sido, hay que tener en cuenta el problema de la falta de registro para su estudio. Puesto que la mayoría de estas personas no desarrollan una 'migración', entendido como un desplazamiento definitivo, o temporalmente largo, sino que pasan temporadas en su otra casa, generalmente no se empadronan en el lugar (municipio) de destino, lo cual dificulta enormemente el estudio de este fenómeno.

Para conseguir los objetivos planteados se ha utilizado una metodología de carácter cualitativo, teniendo en cuenta la importancia que las perspectivas cualitativas han adquirido en los últimos años en el estudio de las migraciones (Borkert et al., 2006); se han realizado entrevistas en profundidad semi-estructuradas que contenían preguntas dirigidas y abiertas, y que han sido contestadas con total libertad y sin control de tiempo por parte de los entrevistados; de esta forma se da la palabra a los actores para que nos enseñen su realidad, que, en ocasiones, no coincide con la que emerge de la interpretación de los datos cuantitativos. El método utilizado ha sido el análisis temático, que ha implicado, primero, el análisis descriptivo de los comentarios, y después la interpretación y conceptualización de la información.

El número de entrevistas realizadas (entre diciembre de 2006 y septiembre de 2007) ha sido de 29 , las cuales han formado parte de un estudio más amplio sobre las estrategias residenciales de los mayores ${ }^{1}$. Los entrevistados han sido personas jubiladas en su mayoría (mayores de 65 años y que habían desarrollado alguna actividad económica remunerada), y un número menor de personas prejubiladas (con menos de 65 años). Todos ellos, después de haber residido en la ciudad de Madrid o en su área metropolitana más próxima, han desarrollado, en un momento próximo a la jubilación, algún tipo de movilidad residencial (desde Madrid) hacia otros lugares del país (zonas costeras y de interior, rurales y urbanas) en donde poseían una segunda vivienda en propiedad.

La elección de Madrid como lugar donde realizar el trabajo de campo responde a que esta región ocupa el tercer puesto en población absoluta con 65 y más años en España (datos del INE), y junto con Andalucía y Cataluña, las tres concentran casi el $43 \%$ de la población mayor del país; por tanto, la gran concentración de personas mayores, unido al hecho de estar residiendo en un

${ }^{1}$ El estudio se ha realizado con financiación aportada por la Fundación MAPFRE (convocatoria 2005), y también en el marco del proyecto 'Cambios residenciales, envejecimiento saludable y mejora de la calidad de vida en la vejez', financiado por el IMSERSO (Referencia 46/2006). 
entorno urbano de gran tamaño, nos sitúa en un excelente laboratorio para el tratamiento de un fenómeno social como la movilidad residencial.

Los lugares de destino son municipios de la costa mediterránea (16 entrevistados) y sólo uno en la costa asturiana (mapa 1); les siguen quienes se han trasladado a municipios rurales de alrededor de Madrid, principalmente localizados en la Sierra (6) y otros lugares (2); finalmente, otras personas han desarrollado esa movilidad hacia otras zonas del interior del país (5).

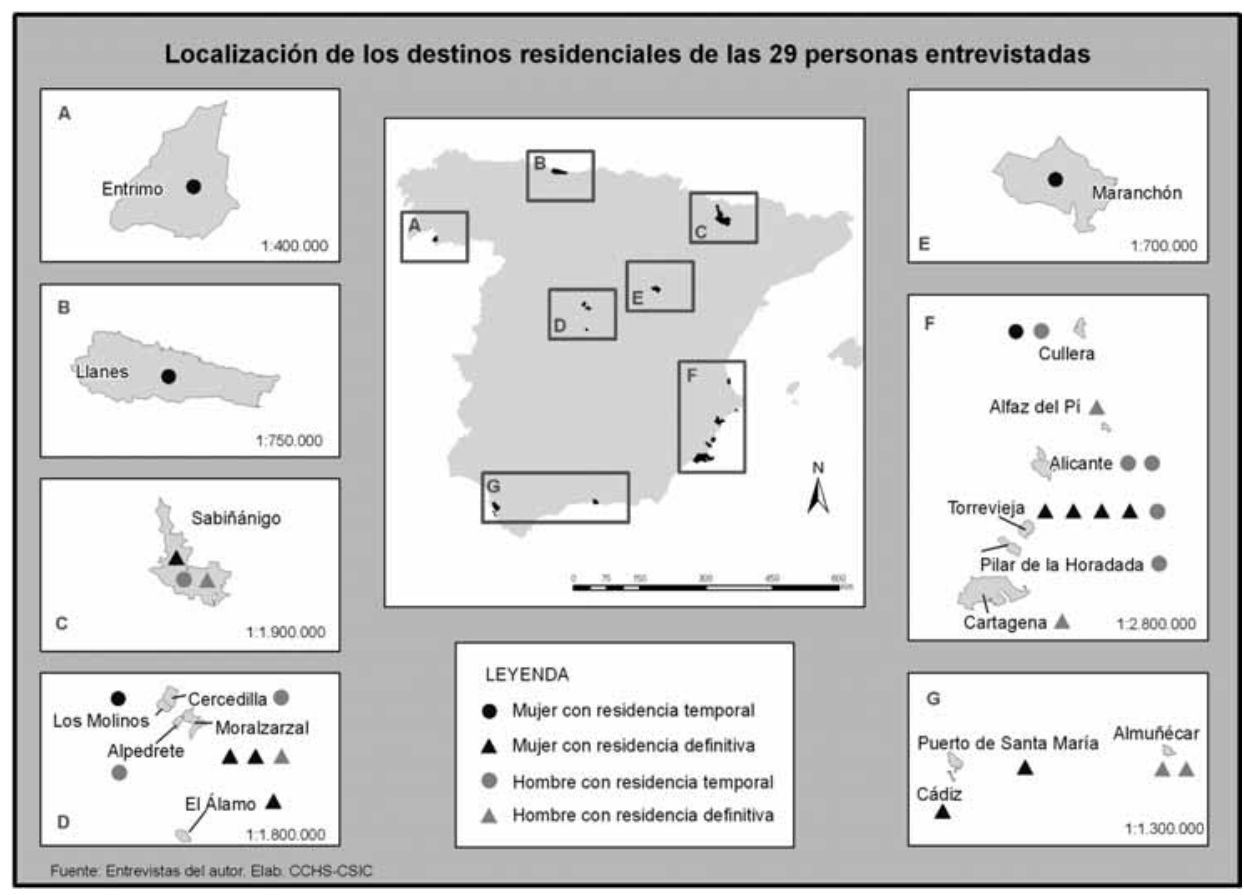

De los 29 entrevistados, 15 son mujeres y 22 tienen 65 años o más, por lo que ya están jubilados (todos habían tenido actividad económica previa); a su vez, 10 personas están entre los 70 y 74 años. Atendiendo a su estado civil, 17 de ellos están casados (de los cuales 16 tienen hijos, unos pocos de los cuales todavía viven con ellos en la vivienda del progenitor), 6 son solteros, 3 viudos y 3 separados. Del total, 12 se han trasladado definitivamente a su nueva residencia, mientras que los otros 17 van y vienen a Madrid, pasando temporadas en cada una de sus viviendas (el requisito para poder habar de cambio residencial es que pasen, al menos, de tres a cuatro meses al año en el lugar donde tienen la residencia secundaria). 
Sin embargo, conviene tener presente algunas peculiaridades de la muestra y del colectivo que se analiza. Este fenómeno de la movilidad se asocia con un cierto poder adquisitivo (son personas que ya disponían de una segunda vivienda, bien sea comprada por ellos o cedida/heredada), por lo que integran una clase social media amplia. La elección de personas para entrevistar con segundas residencias en propiedad responde al factor detonante de movilidad residencial que tienen ese tipo de viviendas y a la conocida importancia que las primeras visitas en vacaciones ejercen como derivación hacia movilidad más sostenida a partir de la jubilación (Flognfeldt, 2002; Müller, 2002). Sobre este colectivo de personas y el tipo de movilidad residencial que desarrollan (amenity led migration) tampoco hay que olvidar que se produce entre personas todavía relativamente jóvenes, que el cambio residencial ha sido elegido libremente, y que estas personas se encuentran en un momento de su vida en el que la finalización de sus ataduras y compromisos profesionales les deja mucho tiempo libre.

\section{EL CONTEXTO DE LA MOVILIDAD RESIDENCIAL: FACTORES INFLUYENTES}

Detrás de la movilidad residencial de los mayores existen determinados factores macro, estructurales, que la impulsan y favorecen, básicamente de tipo social, económico o cultural. Demográficamente, el gran cambio que se está produciendo actualmente en las sociedades desarrolladas, incluida la española, es el envejecimiento de la población, que supone el crecimiento (absoluto y relativo) de las personas mayores (Rojo y Fernández-Mayoralas, 2002) y cuya manifestación espacial da lugar, en ocasiones, a elevadas concentraciones de personas de más edad, como en el caso de Madrid (López, 1993). Teniendo esto en consideración, el potencial de movilidad residencial en personas mayores desde grandes ciudades como Madrid es elevado.

Otros factores favorecedores de la movilidad es que los mayores cada vez llegan a la jubilación con mejor nivel de estudios y en mejor situación económica que en el pasado (Bosch Font, 1995), además de que se jubilan (prejubilan) a edades cada vez más tempranas. A ello hay que añadir las implicaciones de la emancipación de los hijos, que los coloca en una situación de liberación familiar y de desligarse de ataduras familiares; se trata de factores que permiten afrontar la vejez en mejores condiciones y adoptar nuevos comportamientos. La generación de españoles que está llegando hoy en día a la jubilación puede vivir la vejez de manera más activa y menos estigmatizada, y en unas condiciones y con una independencia que nunca antes habían logrado estas 
personas; también la mayor participación e inserción social las coloca en una situación que nunca antes tuvieron sus antecesores (Sánchez Vera, 2002).

Pero además de estos factores, tenidos en cuenta como 'telón de fondo' de la movilidad residencial de los mayores, hay que conocer las razones concretas que impulsan a los mayores a cambiar su lugar de residencia. Se trata de razones que pueden ser muy diferentes de las que actúan de detonante de otras estrategias residenciales desarrolladas durante la juventud y en edades adultas (Puga, 2001; Puga, 2004a).

Si se acota la movilidad residencial a aquélla producida únicamente por razones de amenidad, las razones encontradas por los entrevistados para cambiar su lugar de residencia no varían respecto a las que se han hallado en estudios similares (Casado-Díaz et al., 2004; Duncombe et al., 2001); así, un clima mejor y, en ocasiones, la cercanía del mar, mayor tranquilidad, menor coste de la vida (incluyendo el precio de la vivienda), impuestos más bajos, acceso a bienes y servicios culturales, turísticos y de amenidad, la proximidad de servicios sanitarios, han sido las motivaciones más comunes para el traslado residencial (Lardiés, 2008). No obstante, las razones varían en función de si la movilidad se realiza a escala nacional o internacional, o en un contexto/ámbito geográfico u otro (como el distinto nivel de criminalidad, más o menos inversión en distintas políticas, o determinados factores fiscales).

En esa línea, en esta investigación se ha visto que los principales factores que han ayudado a tomar la decisión del cambio residencial tienen que ver con la búsqueda de tranquilidad y con las condiciones ambientales de Madrid y del nuevo destino (expresadas a través del paisaje, bien sea de mar o montaña), pero también con la cercanía a la familia, con la distancia entre Madrid y la nueva casa (sobre todo si todavía queda familia en Madrid), con las características (equipamiento) de las viviendas, y con la condiciones de vida en el lugar de destino.

\section{Poseer una segunda residencia y la influencia del lugar de nacimiento}

La importancia del ocio y de la práctica de actividades durante la vejez está suficientemente documentada (Neal et al., 1999), y diversos estudios empíricos han mostrado los enormes beneficios de la realización de actividades de ocio, como una importante fuente subjetiva de bienestar (Onishi et al., 2006). En ese sentido, de la misma forma que la vivienda principal es un elemento que da seguridad y tranquilidad (Rojo y Fernández-Mayoralas, 2002), la tenencia de una segunda vivienda se asocia a la práctica de actividades de ocio y 
al tiempo libre, sobre todo en una etapa de la vida en que las obligaciones laborales se han terminado.

Por tanto, se debe considerar la tenencia previa de una segunda residencia entre los mayores como un elemento que favorece, en ocasiones, el desarrollo de una estrategia residencial después de la jubilación. Antes de la jubilación, normalmente se acude a ella durante fines de semana y en vacaciones, o incluso en largas temporadas dependiendo de la cercanía a la que se encuentra del lugar de residencia habitual; sin embargo, después de la jubilación, esta vivienda se convierte en un reclamo para implementar la calidad de vida de estas personas, desencadenando muchas veces un traslado residencial.

En esta investigación, se ha observado que la estrategia de movilidad residencial no siempre es premeditada ni está programada con mucho tiempo antes de la jubilación, sino que se produce de forma bastante espontánea y en función de diversos factores sobrevenidos. El desplazamiento ha venido condicionado por la existencia de la segunda residencia, a la que la mayoría de los entrevistados acudían desde hace varios años antes de jubilarse. Veintiuno de los veintinueve entrevistados adquirieron la vivienda secundaria que ahora utilizan a lo largo de su vida laboral para poder disfrutarla en vacaciones; lo más habitual es que se adquiera entre cinco y diez años antes la jubilación, y sólo unos pocos lo han hecho con veinte o treinta años de antelación. Únicamente en tres casos las casas fueron heredadas de sus padres, y dos de estas personas forman un matrimonio, por lo que esas dos personas se han juntado con dos viviendas en el momento de su jubilación; se utilizan las dos, aunque lo habitual es pasar más tiempo al año en una que en otra.

Con el fin de considerar el potencial de movilidad de la población mayor en la Comunidad de Madrid, a continuación se analiza el número y localización de segundas viviendas en manos de población mayor residente en el municipio y en la Comunidad Autónoma de Madrid. Para ello se han utilizado los microdatos correspondientes al Censo de la Población (2001).

Casi el 28\% (213.000) de las personas de 65 y más años censadas en la región poseían una vivienda secundaria, si bien ese porcentaje es ligeramente superior entre los mayores residentes en el municipio de Madrid (31\%), que es donde se concentra la mayoría de estas personas que disponen de residencia secundaria (casi 170.000). Resulta llamativo que esa cifra es también superior a la que corresponde a la media de hogares de la Comunidad de Madrid. A pesar de ser una de las regiones con menos viviendas secundarias, la de Madrid tiene la proporción más elevada de hogares que disponen de una vivienda de este tipo entre las Comunidades españolas (le siguen Aragón y la Comunidad Valenciana), alcanzando el 22\% del total de los hogares ma- 
drileños, lo cual está muy por encima de la media española del 15\% (Leal y Cortés, 2005).

Además de la gran concentración de mayores en el municipio de Madrid, también se da la circunstancia de que tres de cada diez mayores que residen en él poseen este tipo de vivienda. Sin embargo, esa proporción disminuye cuando las personas residen en municipios de menor tamaño demográfico, en donde sólo uno de cada diez poseen una vivienda secundaria. También es notoria que la tendencia a tener estas viviendas es mayor entre personas con estudios universitarios (el $45 \%$ del total).

Entre los mayores de 65 años, lo normal es que la proporción de adultosjóvenes con residencia secundaria sea más elevada que entre personas más viejas: un 33\% de las personas comprendidas entre los 65 y 69 años la poseen, pero ese porcentaje decrece al $21 \%$ entre los mayores de 80 años; por ello, el $65 \%$ de todas estas viviendas está en manos de personas entre los 65 y 75 años. Es de suponer que a medida que aumentan las dificultades para mantener y visitar esas viviendas, estas personas se deshacen de ellas y dejan de frecuentarlas.

De todos los que poseen una vivienda secundaria, la mayoría $(74,3 \%)$ la tienen fuera de la Comunidad de Madrid, el 22\% en la misma región, sólo el $3 \%$ en el mismo municipio, y muy pocos en el extranjero (gráfico 1); esta distribución de viviendas secundarias demuestra, por tanto, que la localización central de Madrid favorece la movilidad de media-larga distancia hacia todos los puntos peninsulares, pero la proximidad de la Sierra madrileña la convierte en refugio residencial para muchos madrileños (Abellán y Rojo 1997; Puga, 2004b). Por tanto, la mayoría de quienes poseen una segunda vivienda la tienen localizada en otra comunidad autónoma distinta a Madrid, lo cual puede estar en relación con el lugar de origen de estas personas; además, la capacidad de atracción de la Comunidad de Madrid es limitada, ya que las zonas que no están tocadas por el proceso de urbanización generado por la expansión de la ciudad son restringidas.

Otra cuestión importante que puede actuar de detonante de la movilidad tiene que ver con el lugar de nacimiento de estas personas. Así, es probable que las personas originarias de otros lugares de España y que emigraron a Madrid hace algunas décadas hayan mantenido o adquirido alguna propiedad en sus lugares de origen, en donde pasar sus vacaciones y el tiempo de ocio (Abellán y Rojo, 1997). Si se ordena el número de viviendas secundarias que poseen los mayores madrileños por provincias de destino, tras Madrid (34\% del total) aparece Alicante (10,5\%), seguidas de Toledo (8,6\%), Ávila (7,7\%), Guadalajara $(5,4 \%)$ y Segovia $(4,2 \%)$. Se trata en su mayoría de provincias li- 
GRÁFICO 1

TENENCIA DE SEGUNDA RESIDENCIA POR PARTE DE LOS RESIDENTES MAYORES DEL MUNICIPIO DE MADRID, 2001

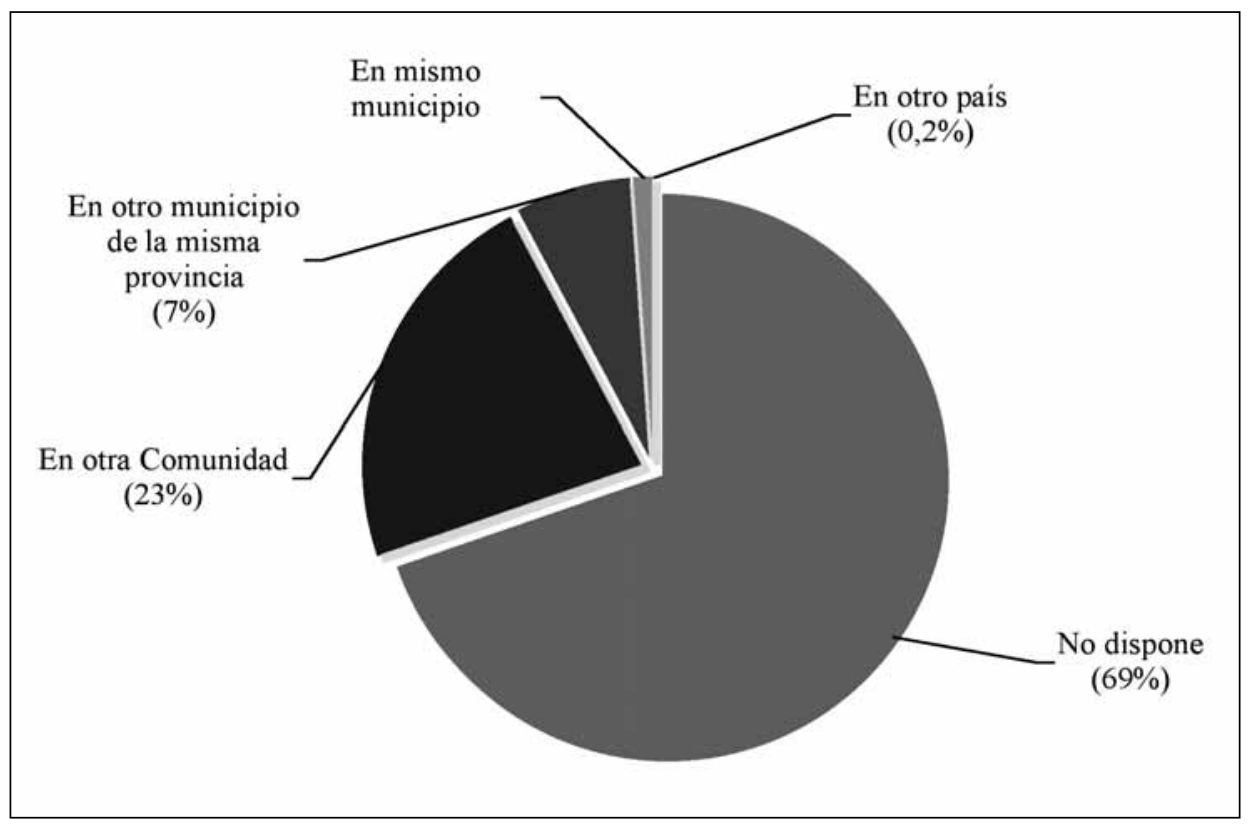

Fuente: Censo de Población, 2001. INE. Elaboración propia.

mítrofes, lo que muestra que frente a lo que podría suponer, no domina la vivienda secundaria de playa, por lo que la proximidad es uno de los mayores atractivos en su elección; a la par, también se aprecia la importancia de la vivienda rural, que es la que más abunda en estas provincias cercanas, como un tipo de vivienda secundaria.

Por tanto, parece existir cierta relación entre el lugar o provincia de nacimiento, la tenencia de residencia secundaria y el lugar donde ésta se localiza. Si se presta atención a las comunidades autónomas de nacimiento de estos mayores, son un claro reflejo de la fuerte inmigración vivida en la región madrileña. De los mayores actuales, sólo un 34\% ha nacido en la Comunidad de Madrid, mientras que otras CC.AA. de alrededor, pero también del sur de España, fueron abastecedoras de mucha población en aquellos años (gráfico 2). 
GRÁFICO 2

LUGAR DE NACIMIENTO DE LA POBLACIÓN DE 65 Y MÁS AÑOS RESIDENTE EN LA COMUNIDAD DE MADRID, 2001

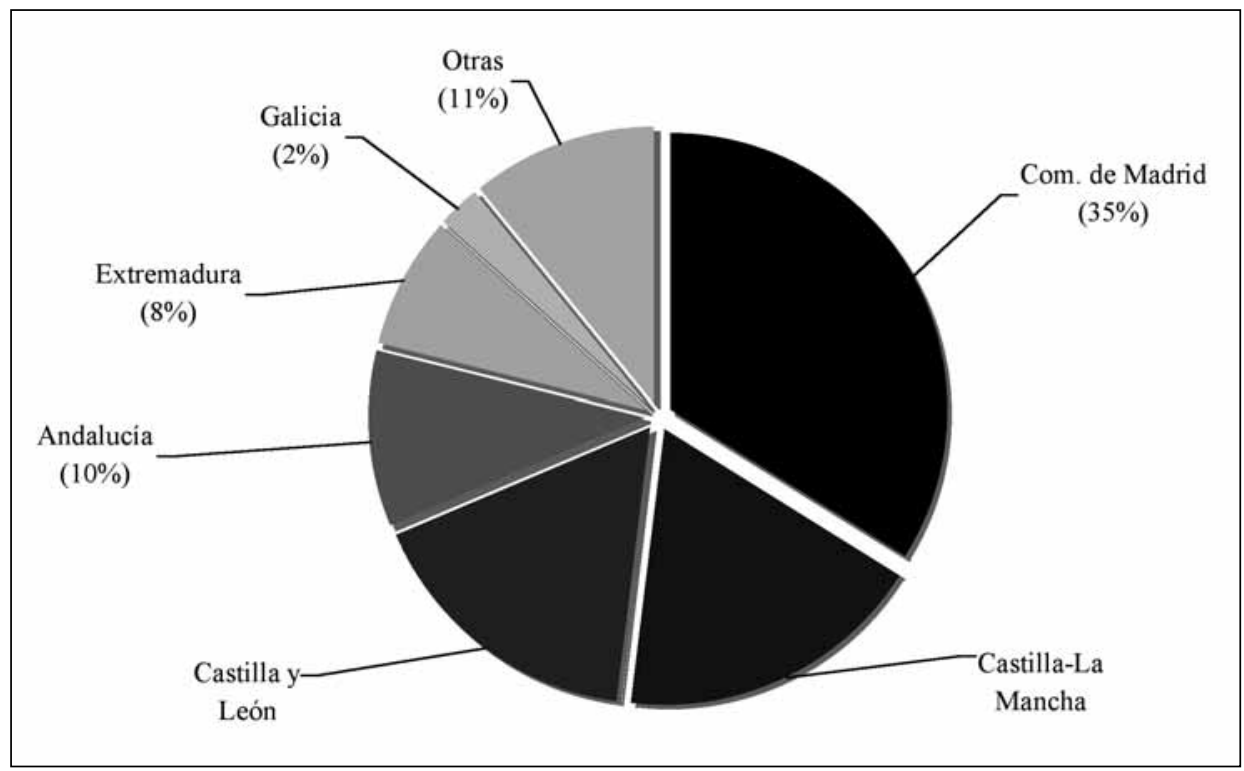

Fuente: Censo de Población, 2001. INE. Elaboración propia.

Paralelamente, si se atiende al tamaño de los lugares de nacimiento de los mayores de la región, el porcentaje más elevado (34\%) ha nacido en grandes ciudades, pero un $20 \%$ procede de municipios menores de 1.000 habitantes y otro $28 \%$ en los comprendidos entre 1.000 y 10.000 . Este origen en lugares de escaso tamaño demográfico hace muy probable que se haya conservado o adquirido alguna propiedad en ellos y que se haya utilizado como vivienda secundaria, pasando en ella, incluso, temporadas más o menos largas.

También conviene tener en cuenta que la proporción de nacidos fuera de la región es mucho mayor entre la población de más edad $(63,5 \%)$ que entre el total de la población $(29,2 \%)$, según los datos padronales de 2006; la explicación, de nuevo, está en la inmigración que recibió la región desde otras regiones españolas durante los años del desarrollismo español. Este elevado número de personas mayores originarias de fuera de Madrid, al igual que la tenencia de residencias secundarias, predispone a estas personas hacia hipotéti- 
cos escenarios de movilidad residencial tras la jubilación, teniendo en cuenta que mucha población mayor posiblemente retorne a su lugar de nacimiento (origen) tras finalizar su etapa laborar en Madrid, o se traslade a residir definitivamente a lo que era su vivienda secundaria (Puga, 2004b); sin embargo, ese análisis excede los objetivos de este trabajo.

\section{Razones ambientales para la movilidad}

Como se ha indicado, años antes del traslado residencial se produjo la compra de la segunda vivienda; la elección de ese lugar responde a que se conocía por haber sido un lugar de vacaciones; en él se habían pasado temporadas, bien en pareja o en casa de amigos, por lo que se cumple nuevamente la conexión entre turismo y migración (Flognfeldt, 2002; Müller, 2002). También el papel de la familia y los amigos para conocer el destino es relevante a la hora de elegir el lugar donde se adquirirá la segunda vivienda. Tan sólo dos personas (solteras) optaron por la compra en el mismo momento de la jubilación o pocos años después sin haber visitado previamente el lugar, y después de conocerlo únicamente gracias a la visita realizada a algún amigo o familiar que ya residía allí.

Tras años de posesión de esa segunda vivienda, las razones ambientales y la tranquilidad en los núcleos de escaso tamaño demográfico donde se han adquirido son destacadas por todos los entrevistados, sobre todo si las características ambientales del nuevo destino se comparan con las de Madrid. La cercanía del mar y lo que ello implica, es el factor de atracción señalado como más importante por la mayoría de las personas con vivienda en zonas costeras; en los otros casos, la montaña, o la cercanía a ella para realizar excursiones, su paz y su tranquilidad, son también los factores más valorados para emprender el traslado. El Sr. E., de 60 años, separado, opina sobre la elección de su lugar de retiro en el sur de España:

«Quería vivir cerca del mar, en la costa, yo soy andaluz, e hice una prospección por toda la costa andaluza y decidí que ésta era la que más me gustaba, y me la compré aquí. De la zona me gusta el mar, quería estar en Andalucía, pero por el clima, porque se vive muy bien todo el año; el clima, por supuesto, también la gente, y las condiciones de vida. Mis padres eran de Huelva, y siempre en verano iba a allí, pero aquello no es un sitio bueno donde se pueda vivir en invierno, porque es más salvaje. En cambio, en esta parte de Granada hay unas infraestructuras donde se puede vivir muy bien todo el año; miré toda esta parte de Málaga, Almería y Granada, y esta es la zona que me encantó». 
A la Sra. P., casada, de 64 años, le gusta de su nuevo lugar de residencia la gente y la tranquilidad:

«Es otra vida. Es otra vida totalmente diferente. Socialmente, el entorno, la naturaleza, no tiene nada que ver. En Madrid no hablas con nadie, y aquí hablo con todo el mundo porque conoces a todo el mundo. A la gente la conocíamos desde hace años, de cuando veníamos, aunque ahora la hemos ampliado, hasta con los del mismo pueblo, porque tenemos un contacto casi a diario. Y luego la vida aquí..., que no tiene nada que ver; te vas con el coche a tres kilómetros, a Navacerrada, la jara, el tomillo, es que esto no tiene nada que ver; el entorno es diferente».

Sin embargo, esas condiciones ambientales que actúan como factores de atracción durante buena parte del año, otras veces se convierten en factores repelentes, en relación a la gran afluencia de turistas y población que provocan durante el verano, o también a las altas temperaturas que se registran en los lugares costeros. Salvo para unos pocos entrevistados que manifiestan ir menos a la vivienda secundaria conforme pasa el tiempo, el resto de personas están contentas con la elección del lugar realizado, aunque casi todas las que tienen localizada su vivienda en lugares costeros aluden a la congestión y masificación veraniega y a los problemas de tráfico como justificación para evitar el destino durante los meses centrales del verano. Durante esas fechas ceden la residencia a familiares (hijos, generalmente), que son los que pueden hacer uso de la casa por tener que disfrutar las vacaciones necesariamente en verano. Es entonces cuando los entrevistados aprovechan para pasar una temporada en Madrid y estar en contacto con familiares y amigos, además de utilizar la oferta cultural que la capital ofrece.

Madrid genera muchos elementos de rechazo que actúan como razones para que muchas personas la abandonen. No siempre es posible hacerlo, pero la amenaza que en algunas personas genera la población inmigrante, un cierto desorden en la ciudad, y la inseguridad, son razones que algunos entrevistados como este jubilado, casado y con 73 años, dan para cambiar su lugar de residencia:

«Antes nos conocíamos todos en donde yo vivo, era una zona tranquila. Era un barrio nuevo, todo con gente joven. Cuando me jubilé me quería ir a otro sitio, pero era prácticamente imposible por los precios que tenían los pisos. Además, hoy en día ha cambiado todo. Ahora ya no hay respecto a nada ni nadie, además viene gente nueva...; yo no quiero ser racista, pero te obligan a serlo. ¡Nadie respeta nada!. Botellas, hasta los carros del supermercado, que los encuentras por la calle... Además, tampoco hay seguridad como había antes. Así que uno quiere marcharse, pero no puede, y como no te puedes ir a otro sitio, pues te vas al pueblo». 
Relacionado con la búsqueda de tranquilidad y con la demanda de determinadas condiciones ambientales está el tamaño demográfico de los núcleos de población del lugar de destino; se trata, por lo general, de núcleos de medio o pequeño tamaño; muchas veces son pueblos de no más de 10.000 habitantes (los costeros), aunque algunos de ellos también son de menor tamaño (generalmente los de interior). Sólo dos de las personas entrevistadas poseen su vivienda en las ciudades de Alicante y Murcia, siendo las únicas, por tanto, que se han trasladado a un núcleo urbano de mayor tamaño demográfico.

Algo diferente es que en el lugar de destino residieran familiares o amigos y eso haya impulsado a estas personas al traslado residencial; esto sólo ha ocurrido en cuatro casos, ya que la mayoría de estas personas no tienen ni familiares ni amigos residiendo en el nuevo lugar; por tanto, pocas personas tenían conocidos en el nuevo destino, pero tenerlos, no ha sido la motivación más importante para plantear la movilidad.

\section{La cercanía a la familia}

Estudios previos señalan la importancia de determinados acontecimientos familiares a la hora de generar distintos tipos de movilidad residencial en la vejez (Puga, 2004b), como el que los hijos abandonen el hogar, que la red familiar se reduzca, o que también lo haga la red social en torno a los individuos. Como se indicará a continuación, los factores familiares son importantes a la hora de desencadenar movilidad residencial, una vez que estas personas se sienten liberados de las 'ataduras' de los hijos.

También las personas mayores suelen mantener lazos muy estrechos con sus familiares, y viceversa; es algo que Rosemayer y Kockels han definido como el mantenimiento de la «intimidad a distancia» (Walker, 1996). Investigaciones realizadas en diferentes países europeos confirman los contactos frecuentes que se mantienen entre las personas mayores y sus familiares. España es el país europeos donde con más frecuencia se tiene contacto diariamente con otros miembros de la familia: el $60,7 \%$ de los mayores ve diariamente a otros miembros, seguido por el 15,5\%, que los ve dos o más veces por semana (Walker, 1996). Por este motivo, el contacto con la familia debe ser relacionado con la distancia a la cual se traslada la residencia, debido a las diferencias encontradas en los motivos de migración según la distancia de los desplazamientos (Da Vanzo, 1982).

Así pues, la distancia entre Madrid y el lugar donde inicialmente se adquiere la segunda vivienda es importante a la hora de determinar la frecuenta- 
ción y el número de veces que se puede ir y venir a lo largo del mes o del año, tanto si todavía se trabaja o ya se está jubilado y la persona se plantea visitar la segunda vivienda con más o menos asiduidad e incluso el traslado residencial definitivo. La existencia de familia, el contacto mantenido y la distancia (medida en tiempo) entre ambos lugares, y en consecuencia el posible futuro lugar de retiro, son factores que están muy presentes y son importantes para la mayoría de las personas:

«La edad influye, porque si tienes menos tiempo y vas los fines de semana y tú piensas que vas a disfrutar a 40 kilómetros todos los viernes después de salir de trabajar y disfrutas del fin de semana y entonces, cuando estás en activo y tienes una perspectiva de vida y tienes los niños pequeños, entonces, lógicamente, tendemos a comprar más próximo a la capital que es donde vivimos» (Sra. M.L., casada, de 71 años, jubilada).

A mayor edad de la persona, la distancia que hay que recorrer es especialmente importante si todavía hay hijos que se quedan residiendo en Madrid, y sobre todo si hay nietos (factor que ejerce más influencia para las mujeres); en cambio, esta influencia es menor entre solteros o personas sin descendencia. Así, algunos que se han trasladado a lugares alejados no más de 100 kilómetros de Madrid admiten que no se hubieran ido a residir más lejos porque entonces no hubieran podido estar cerca de los hijos y nietos. También la distancia influye en que el traslado sea temporal o tenga un carácter definitivo, aunque en ello también tienen que ver otros factores como la estrecha relación que todavía se pueda mantener con Madrid (como lugar de importantes ofertas culturales, y por otras razones de tipo social, sanitario y climático). En cuanto a los factores distancia y tamaño demográfico del núcleo de destino, por lo general, el análisis de la distancia recorrida y el tamaño de los lugares de procedencia y destino nos lleva a conclusiones parecidas a las halladas en los tradicionales estudios sobre migraciones en los que se han aplicado modelos gravitacionales (Conway y Houtenville, 2003).

Lo anterior introduce, a su vez, pautas de movilidad distintas según el estado civil, ya que por lo general, la mayoría de los entrevistados están casados, lo cual demuestra que la movilidad residencial se realiza fundamentalmente en pareja; este hecho corrobora lo señalado por Puga (2004a), al considerar que el proyecto migratorio es un asunto de pareja. Las parejas de casados demuestran generalmente mayor propensión que los no casados a emigrar a distancias mayores por motivos de amenidad, así como a sustituirlos por traslados residenciales temporales (Warnes, 1992). 
Sin embargo, ese comportamiento desarrollado por los casados se contradice con los mostrados en otros estudios, en donde se afirma que las personas solteras y viudas muestran mayor movilidad residencial. Así, López (2004) corrobora que las tasas de movilidad residencial de los viudos son ligeramente superiores a la del resto de individuos de la misma edad en todos los países europeos, a excepción de lo que ocurre en Finlandia y Alemania. También Northcott (1985) demuestra para Canadá que las personas viudas y divorciadas eran más móviles que los que estaban casados. En ambos casos, parece ser que la movilidad asociada a la pérdida del cónyuge provoca en la mayoría de los países un aumento de la movilidad residencial.

Teniendo en cuenta lo anterior, parece no existir una pauta de movilidad generalizable respecto a la personas viudas, ya que el efecto de la viudedad en las personas puede variar según la edad a la que ocurre este acontecimiento, la situación en la que se encuentra la persona, y dependiendo del momento o situación en la que se produce la muerte de la pareja. Por un lado, a mayor edad de la persona viuda aumenta la probabilidad de que esa persona ya no pueda vivir sola, requiera atención, así como la adaptación a una vivienda de menores dimensiones, todo lo cual puede motivar un traslado residencial en esa nueva etapa vital. Sin embargo, y entre personas más jóvenes y en mejor estado de salud, la muerte del cónyuge suele conducir a quien enviuda a un momento duro y doloroso, que frena cualquier iniciativa de movilidad y de irse a vivir a otro lugar. Éste, al menos, es el sentimiento expresado por algunos de los entrevistados que habían enviudado no hace mucho tiempo.

\section{Dotaciones, equipamientos y servicios}

Otras razones también muy comentadas por más de la mitad de los entrevistados para no pasar la mayor parte del año en el nuevo destino son las relacionadas con la inexistencia de equipamientos y servicios (sobre todo sanitarios, pero también comerciales y culturales o de ocio en general), y con las condiciones de habitabilidad en que se encuentra la vivienda. Investigaciones previas han señalado que, en comparación con los migrantes más jóvenes, las amenidades, entendidas como la existencia de infraestructuras y servicios que ayudan a las práctica de actividades de ocio, son un factor importante en la movilidad de los mayores (Clark and Hunter, 1992; Conway y Houtenville, 2003; Millington, 2000). En concreto, se está de acuerdo en la gran influencia y determinación que la necesidad de servicios médicos y de apoyo en la vejez tienen en la decisión de migrar (Sommers y Rowell, 1992). 
La existencia de hospitales y la cobertura sanitaria es un factor muy importante como determinante de la movilidad residencial, tal y como se ha señalado en diversos estudios realizados tanto en el ámbito norteamericano (Kallan, 1993) como en el europeo (Casado-Díaz et al., 2004). Entre los entrevistados para este trabajo no se ha hecho alusión al gasto en sanidad como factor-razón de movilidad, pero sí a la existencia o no de centros de asistencia sanitaria (centros de salud y hospitales) y a la distancia a la que se encuentran.

Querer sentirse cerca de hospitales, sobre todo en personas que han tenido problemas médicos importantes y están sujetos a tratamientos de salud en Madrid, actúa de freno para el cambio definitivo del lugar de residencia, aunque parece ser un aspecto más importante en mujeres que en hombres. También esta razón tiene que ver con que si la persona abandona Madrid temporalmente, cuando visite a un médico, tendrá que explicarle nuevamente su historial, en el lugar de destino, lo cual genera cierto rechazo para la utilización de estos servicios. Eso hace que en muchos casos, cuando se ha producido un traslado a zonas no muy alejadas de Madrid, todavía se sigue visitando a los médicos de siempre; eso ocurre cuando el destino está a una distancia no mayor de 100-120 $\mathrm{km}$ de Madrid, pero cuando es más, se aprovecha igualmente para visitar a médicos en Madrid, continuar los tratamientos iniciados antes del traslado, a la vez que se visita a la familia, o se hacen gestiones en la ciudad. Un ejemplo de esto es la Sra. P. (casada, de 64 años, que reside actualmente en un pueblo a $50 \mathrm{~km}$ de Madrid, y que tiene una hija casada que vive en Madrid):

«Normalmente bajamos a Madrid los jueves, todo el jueves, y bajamos todos, a ver mi nieto, que vaya, para mí es... lo primordial [...]. A Madrid seguimos yendo por mi hija y el nieto, pero también por el tema de médicos. Mi marido ha tenido dos cánceres; un melanoma, cogido a tiempo, y un tumor de próstata que se lo han quitado, y tiene que seguir haciéndose revisiones».

Otra opinión distinta es la de personas que habiéndose ido definitivamente (o no necesariamente), opinan que actualmente la asistencia sanitaria ha mejorado mucho en todas las zonas de España (especialmente en las rurales) y que hoy en día se puede tardar menos tiempo en llegar a un hospital viviendo fuera de Madrid que en la capital.

También son bastante citados otros dos factores para no querer abandonar definitivamente Madrid. El primero tiene que ver con la mayor oferta de todo tipo que la ciudad ofrece (en cuestión de servicios, comercios y actividades de ocio y lúdicas). En este sentido, prácticamente la mitad de los diecisiete entrevistados que todavía pasan algunos meses al año en Madrid aluden a la cantidad de actividades, oferta cultural, y en general cosas que hacer que ofrece la ciudad. 
«En verano estoy en Madrid porque me gustan los actos culturales: teatro, zarzuelas, las fiestas de Madrid, salir por las noches... y por eso estoy en agosto y vamos al teatro, a festivales, etc.» (Sr. A., casado, con 74 años, que reside temporalmente en Alicante).

Otro factor que ha posibilitado o frenado la movilidad ha sido la posible venta o alquiler de la anterior vivienda que se utilizaba en Madrid. La propiedad de la vivienda ejerce un fuerte vínculo con el territorio, reteniendo a la población y reduciendo las probabilidades de migrar, también en la vejez. Se conoce que la movilidad declina entre quienes son propietarios de su vivienda (Sommers y Rowell, 1992). Sin embargo, este aspecto no puede llegar a ser investigado satisfactoriamente en este trabajo debido al tipo de personas elegidas como objetivo de estudio (siendo propietarios de una viviendas secundaria y sólo personas que han desarrollado movilidad residencial), lo que impide conocer qué ocurre entre quienes no han decidido emigrar o cambiar su lugar de residencia. Sin embargo, sí se ha podido comprobar la importancia de la propiedad de la vivienda utilizada en Madrid, puesto que muy pocas personas deciden deshacerse de ella; lo más habitual es alquilarla o cederla a los hijos, y sólo en casos muy extremos (por necesidad económica, pero en muy pocos casos) se ha llegado a vender.

Otra razón que afecta al traslado definitivo hacia el lugar donde se localiza la segunda residencia hace referencia al mejor equipamiento de la casa de Madrid (principalmente en calefacción y agua caliente), ya que algunos de los entrevistados no han mejorado el equipamiento de sus casas (en el nuevo destino) y éstas siguen con las mismas dotaciones que el día que empezaron a ser usadas para pasar básicamente unos días de vacaciones en verano. Se trata de viviendas localizadas o bien en la costa mediterránea o en zonas de interior o de montaña, por lo que en ambos casos los entrevistados señalan que durante el invierno (días en los que los interiores de las viviendas en zonas del Mediterráneo, por ejemplo, están frías sin calefacción) se sienten mejor en sus casas de Madrid. Pasar el invierno en la otra casa supondría en ocasiones duplicar gastos, cuando la calefacción central está incluida muchas veces en los gastos de comunidad en la vivienda de Madrid.

\section{Otros factores}

El hecho de haber residido durante mucho tiempo en Madrid o haber nacido incluso en la ciudad, introduce el tema raíces como argumento para irse a vivir a otro lugar o no. Este aspecto emerge en personas que en el momento de 
la jubilación no se podrían acostumbrar a pasar largas temporadas fuera de lo que esta ciudad ofrece. Esta idea aparece aproximadamente en más de la mitad de las personas entrevistadas que no contemplan irse definitivamente a vivir al lugar donde tienen la segunda residencia.

El coste de la vida es otro de los factores que normalmente más condiciona e influye en la decisión de abandonar un lugar y elegir otro para el retiro (Kallan, 1993). Este factor, que se ha demostrado influyente en los desplazamientos de retiro de las personas mayores, no parece haber influido entre los entrevistados que se han desplazado desde la ciudad de Madrid, lo cual tiene sentido debido al aumento pero también a la homogeneización general de los precios en España, sobre todo entre ciudades de mayor y menor tamaño demográfico, y entre zonas urbanas y rurales. Sobre esto, algunas personas han manifestado que no notan diferencias de precios entre Madrid y los lugares elegidos para pasar su retiro, y que son inexistentes, puesto que los artículos de primera necesidad los compran en el mismo tipo de supermercados. De la misma forma, otros factores que en algunos países llegan a tener sentido, como las diferencias de impuestos y tasas, en inversiones y gasto público, y de tipo fiscal (Clark y Hunter, 1992), no han aparecido entre los entrevistados, lógicamente porque al tratarse de una movilidad residencial a escala nacional en muchas ocasiones esas diferencias no existen o son inapreciables.

Teniendo en cuenta cómo afectan todos estos factores para emprender un cambio residencial definitivo, muy pocas personas que todavía residen parte del año en Madrid están dispuestas a emprenderlo. Sólo una persona declara que se irá a residir a Alicante cuando su hijo se independice y deje de vivir con ellos, y otra dice que tiene previsto pasar más tiempo en su segunda residencia y menos en Madrid, pero a pesar de reconocer que Madrid sigue siendo un punto de referencia para realizar actividades culturales y ver a amigos y solucionar asuntos particulares. El resto lleva intención de seguir entre las dos viviendas (parte del año en cada una). Parece que la libertad de ir y venir, pasando temporadas en cada una de las viviendas, es lo que más valoran estas personas, siempre y cuando lo puedan hacer sin ningún tipo de ataduras ni obligaciones.

\section{CONCLUSIONES}

Las migraciones internas y la movilidad residencial en España han sido, y todavía constituyen, un factor importante en la evolución y distribución de la población, siendo un fenómeno socialmente muy significativo. Al impacto demográfico de los desplazamientos residenciales de población mayor hay que 
añadir otros como el cultural, el económico (sobre todo en zonas rurales y/o despobladas) y el inmobiliario, sin olvidar la mayor o menor demanda que estos desplazamientos generan en la utilización de determinados servicios (sociales, sanitarios ...) (Rogers et al., 1990). No se tiene en cuenta como se debiera, pero la concentración de personas mayores en determinadas áreas tiene una influencia muy nítida en la necesidad (o no) de determinados servicios, viviendas, y en sus características y equipamientos.

Según Willekens (1985), la distribución de la población no se puede explicar sin considerar los motivos y los eventos en el curso de vida relacionados con las decisiones de movilidad-migración. Además, el proceso de la toma de decisión es el determinante más próximo del comportamiento migratorio (De Jong, 1999). Los estudios tradicionales han analizado las migraciones como un fenómeno agregado, pero también los gestores públicos y cada vez más investigadores, movidos por el auge de nuevas tendencias metodológicas, reconocen que para explicar las grandes cifras es necesario conocer cómo las personas, individualmente o en grupo (familia) toman decisiones y evalúan opciones y alternativas que influyen en dónde y cómo vivir.

Este trabajo ha tenido un carácter exploratorio, pero ha permitido descubrir que las razones ambientales, la presión a la que las personas mayores se ven sometidas en una gran ciudad, están detrás del deseo de cambiar el lugar de residencia. Pero esos factores son tan importantes como los de tipo familiar: tanto los hijos como los nietos ejercen una influencia muy notable a la hora de pensar en el desplazamiento y en su duración, aunque más en el caso de las mujeres que de los hombres. La conclusión es que la población de más edad disfruta la libertad de 'moverse' libremente entre Madrid y el nuevo destino residencial, pero de forma temporal, y que la mayoría de estas personas no baraja la posibilidad de emprender un cambio residencial definitivo y para el resto de su vejez; son felices con la idea de ir y venir entre las dos viviendas (la principal en Madrid y la secundaria) sin romper drásticamente con su vida anterior.

A partir de aquí falta todavía por profundizar sobre muchos aspectos relacionados con esta temática. En el futuro habrá que ver cómo y en qué contexto y situaciones se produce la decisión de emigrar, y seguir estudiando de qué depende que esa movilidad se produzca con carácter más o menos definitivo, hacia dónde, relacionando eso con las características de los lugares de origen y de destino. También sería interesante adoptar una perspectiva longitudinal con el fin de saber qué ocurre a medida que pasa el tiempo con personas que en su día emigraron, además de conocer las razones por las cuales otras personas nunca llegan a desplazarse. 
En este contexto entra en juego la metodología cualitativa, como la mejor herramienta para profundizar en las historias sobre la movilidad, los detonantes, actitudes, razones, temores, sentimientos, niveles de satisfacción, etc., difíciles de ser recogidos en un cuestionario con preguntas cerradas o semi-cerradas.

Recibido: 23/10/2008

Aceptado: 29/04/2010

\section{BiBLIOGRAFÍA}

Abellán García, A. y Rojo Pérez, F (1997): "Migración y movilidad residencial de las personas de edad en Madrid". Anales de Geografía de la Universidad Complutense, 17, 175-193.

Borkert, M., Martín, A., Scott, S. y De Tona, C. (2006): "Introducción: Comprender la investigación sobre migraciones (a través de fronteras nacionales y académicas) en Europa" [14 párrafos]. Forum Qualitative Sozialforschung/Forum: Qualitative Social Research, 7(3), Art. 3. [http://www.qualitative-research.net/fqs-texte/3-06/06-3-3s.pdf].

Bosch Font, F. (1995): "Las edades doradas y el comportamiento financiero", en Las actividades económicas de las personas mayores. Seniors Españoles para la Cooperación Técnica, 373-388.

Casado-Díaz, M. A., Kaiser, C. y Warnes, A. M. (2004): "Northern European retired residents in nine southern European areas: characteristics, motivations and adjustment". Ageing and Society, 24, 3, 353-381.

Clark, D. E. y Hunter, W. J. (1992): "The Impact of Economic Opportunity, Amenities and Fiscal Factors in Age-Specific Migration Rates". Journal of Regional Science, vol. 32, núm. 3, 349-365.

Clark, D. E. y Hunter, W. J. (1992): "The impact of economic opportunity, amenities, and fiscal factors on age- specific migration rates". Journal of Regional Science, 32, 3, 349-365.

Conway, K. S. y Houtenville, A. J. (2003): "Out with the Old, In with the Old: A Closer Look at Younger Versus Older Elderly Migration". Social Science Quarterly, 84, 2, 299-328.

Da Vanzo, J. (1982): "Techniques for Analysis of Migration History Data”, en National Migration Surveys, Guideliness for Analyses. New Cork, United Nations, ESCAP, 98-108.

De Jong, G. F. (1999): “Choice Processes in Migration Behavior”, en K. Pandit y S. Davies Withers (eds.): Migration and Restructuring in the United States. A geographic perspective. Boston: Rowmand \& Littlefield Publishers, Inc., 273-294. 
Duncombe, W., Robbins, M. y Wolf, D. A. (2001): "Retire to where? A Discrete Choice Model of Residential Location". International Journal of Population Geography, 7, 4, 281-293.

Flognfeldt, T. (2002): "Second-home ownership: a sustainable semi-migration", en C. M. Hall y A. M. Williams (eds.): Tourism and Migration. Dordrecht, Netherlands: Kluwer, 187-203.

Haas, W. H. y Serow, W. J. (1993): "Amenity Retirement Migration Process: A Model and Preliminary Evidence". The Gerontologist, 33, 2, 212-220.

Kallan, J. E. (1993): "Multilevel Analysis of Elderly Migration". Social Science Quarterly, 74, 2, 403-416.

Lardiés, R. (2008): "Aproximación cualitativa al estudio del efecto de la movilidad residencial en la calidad de vida de los adultos mayores" [74 párrafos]. Forum Qualitative Sozialforschung/Forum: Qualitative Social Research, 9(2), Art. 22. [http://www.qualitative-research.net/fqs-texte/2-08/08-2-22-s.htm].

Leal Maldonado, J. y Cortés Alcalá, L. (2005): Características del parque de viviendas de la Comunidad de Madrid según el Censo de 2001. Instituto de Estadística de la Comunidad de Madrid y Consejería de Economía e Innovación Tecnológica. Madrid.

Litwark, E. y Longino, C. F (1987): "Migration Patterns Among the Elderly: A Developmental Perspective". Gerontologist, 34, 4, 736-756.

López Gay, A. (2004): "Intensidad y calendario de movilidad residencial en la Unión Europea". Scripta Nova. Revista Electrónica de Geografía y Ciencias Sociales, vol. VIII, núm. 174, 1 de octubre. Fecha de consulta: 13-10-2008 [http://www.ub.es/geocrit/sn/sn-174.htm].

López Jiménez, J. J. (1993): El envejecimiento y las personas ancianas en Madrid. Ayuntamiento de Madrid, Área de Servicios Sociales, Madrid.

Meyer, J. W. y Speare, A. (1985): "Distinctively Elderly Mobility: Types and Determinants". Economic Geography, 61, 1, 79-88.

Millington, J. (2000): "Migration and age: the effect of age on sensitivity to migration stimuli". Regional Studies, 34, 6, 521-533.

Módenes, J. A. (2006): "Una visión demográfica de la movilidad residencial reciente en España”. Centro de Estudios Demográficos (CED), núm. 292, 27 p. (Workpaper presentado al seminario "La vivienda en los espacios metropolitanos", Centro de Estudios Andaluces, Sevilla, 27 de septiembre de 2006).

Müller, D. (2002): "German Second homeowners in Sweden: some remarks on the tourism-migration nexus". Revue Européenne des Migrations Internationales, 18, 1 , 67-86.

Neal, J. D. Sirgy, M. J. y Uysal, M. (1999): "The role of satisfaction with leisure travel/tourism services en experiences in satisfaction with leisure life and overall life". Journal of Business Research, 44, 3, 153-163.

Northcott, H. C. (1985): "The geographic mobility of Canada's elderly". Canadian Studies in Population, 12, 12, 183-201. 
Onishi, J.; Masuda, Y.; Suzuki, Y.; Gotoh, T.; Kawamura, T. e Iguchi, A., (2006): "The pleasurable recreational activities among community-dwelling older adults". Archives of Gerontology and Geriatrics, 43, 2, 147-155.

Puga, D. (2001): "Influencia de la biografía migratoria en los movimientos residenciales en la vejez". Anales de Geografía de la Universidad Complutense, 21, 31-52.

Puga, D. (2004a): "El comportamiento residencial de los mayores. Análisis biográfico de la movilidad en la vejez". Revista Española de Investigaciones Sociológicas, 105, 79-102.

Puga González, Ma D. (2004b): Estrategias residenciales de las personas de edad. Movilidad y curso de vida. Barcelona. Fundación "la Caixa".

Rogers, A., Watkins, J. F. y Woodward, J. A., (1990): "Interregional elderly migration and population redistribution in four industrialized countries". Research on Aging, $12,3,251-293$.

Rojo Pérez, F. y Fernández-Mayoralas, G. (eds.) (2002): Envejecer en casa. La satisfacción residencial de los mayores en Madrid como indicador de su calidad de vida. Monografías, 23. Madrid. Consejor Superior de Investigaciones Científicas.

Sánchez Vera, P. (2002): "Sociología de la vejez versus economía de la vejez". Papers: Revista de Sociología, 61, 39-88.

Sommers, D. G. y Rowell, K. R. (1992): "Factors differentiating elderly movers and nonmovers: a longitudinal analysis". Population Research and Policy Review, 11, 3 , 249-262.

Walker, A. (1996): “Actitudes europeas ante el envejecimiento y los personas mayores". Revista Española de Investigaciones Sociológicas, núm. 73, pp. 17-42.

Walters, W. H. (1994): "Place Characteristics in Elderly Migration Research". Bulletin of Bibliography, 51, 4, 341-354.

Walters, W. H. (2002): "Place Characteristics and Later-Life Migration". Research on Aging, 24, 2, 243-277.

Warnes, A. M. (1992): "Age-related variation and temporal change in elderly migration", en A. Rogers (ed.): Elderly migration and population redistribution. London. Belhaven Press, 35-55.

Willekens, F. (1985): Migration and Development: A Micro-Perspective. Voorburg: Netherlands Interuniversity Demographic Institute. Working Paper 62.

\section{RESUMEN}

La movilidad residencial es un fenómeno cada vez más habitual entre la población, y en particular en las personas de más edad. La jubilación suele ser un momento en el que cada vez más personas deciden cambiar su lugar de residencia. Se trata de una decisión que responde a factores de tipo social, económico y cultural, principalmente, aunque para su estudio también es necesario profundizar en otras razones de tipo geográfico, ambiental, familiar, social, cultural, etc. En este trabajo se analizan las razones que han impulsado a personas mayores residentes en Madrid a cambiar su lugar 
de residencia, bien de forma temporal o definitiva, para lo cual se ha trabajado con metodología cualitativa. Es necesario conocer bien esas razones, no sólo para planificar los futuros lugares de retiro, además de los servicios y equipamientos que tales desplazamientos requieren, sino también para contribuir a la mejora de la calidad de vida de estas personas.

PAlAbRAS ClAVE: movilidad residencial; personas mayores; migración; razones; Madrid.

\section{Abstract}

Residential mobility is an increasing phenomenon among the population, but in particular among the old people. The retirement is usually a moment in which more and more people decide to change their place of residence. This is a decision which mainly responds to social, economic and cultural factors; nevertheless, it is also necessary for the study of the mobility to deepen in other geographic, environmental, familiar, social and cultural reasons. In this article, reasons that have impelled to old residents living in Madrid to change the place of residence (both in a definitive or temporary way) are analyzed; for this, qualitative methodology has been used. It is necessary to know well those reasons, not only to plan places for retirement in the future, also to plan services and equipment that such displacements require, and for the contribution to the improvement of the quality of life of these people.

KEY WORDS: residential mobility; old people; migration; peasons; Madrid.

\section{RÉSUMÉ}

La mobilité résidentielle est un phénomène chaque fois plus habituel entre la population, et en particulier entre les personnes plus âgées. La retraite est généralement un moment où plus de personnes décident de changer leur lieu de résidence. Il s'agit d'une décision qui répond à facteurs de type social, économique et culturel, principalement, bien que pour son étude il est aussi nécessaire d'approfondir dans d'autres raisons de type géographique, environnemental, familial, social, culturel, etc. Dans ce travail on analyse les raisons qu'ont propulsé à des personnes plus grandes résidantes à Madrid à changer leur place de résidence, bien de manière temporaire ou definitive; pour ceci, on a travaillé avec méthodologie qualitative. Il est nécessaire connaître bien ces raisons, non seulement pour planifier les futurs lieux de retraite, aussi les services et les équipements que ces déplacements requièrent, mais aussi pour contribuer à l'amélioration de la qualité de vie de ces personnes.

MoTs CLÉs: mobilité résidentielle; personnes âgées; migration; raisons; Madrid. 